\title{
Pneumatosis intestinalis in a patient with chronic bronchiectasis
}

\author{
Maya Doumit MSc MD ${ }^{1}$, Nav Saloojee MD FRCPC ${ }^{1}$, Richard Seppala MD²
}

\begin{abstract}
M Doumit, N Saloojee, R Seppala. Pneumatosis intestinalis in a patient with chronic bronchiectasis. Can J Gastroenterol 2008;22(10):847-850.
\end{abstract}

Pneumatosis intestinalis has been described in association with many gastrointestinal tract disorders including infection, ischemia and obstruction. It has also been described in patients with chronic obstructive pulmonary disease, connective tissue disorders, organ transplantation, leukemia and various states of immunodeficiency. In the present paper, the case of a 66-year-old woman with chronic bronchiectasis who subsequently developed pneumatosis intestinalis is described.

Key Words: Chronic bronchiectasis; Pneumatosis intestinalis

Dneumatosis intestinalis (PI) has been described in association with many gastrointestinal tract disorders including infection, ischemia and obstruction. PI has also been described in patients with chronic obstructive pulmonary disease, connective tissue disorders, organ transplantation, leukemia and various states of immunodeficiency (1-4). In the present paper, the case of a patient with chronic bronchiectasis who subsequently developed PI is described.

\section{CASE PRESENTATION}

A 66-year-old woman with a medical history of chronic bronchiectasis, gastroesophageal reflux disease, hypothyroidism and osteoarthritis was referred to the gastrointestinal clinic for iron deficiency anemia and chronic diarrhea.

With respect to her diarrhea, she reported a five-week history of more than 15 loose bowel movements per day. There was no rectal bleeding. She complained of diffuse abdominal bloating and pain, and denied any recent antibiotic use, travel, infectious contact or ingestion of anything that might have been undercooked.

After the onset of her diarrhea, she was admitted to the hospital with some respiratory issues. She required antibiotics. On discharge from the hospital, her diarrhea settled. She was having one to two bowel movements per day.

The patient underwent a gastroscopy, which was normal. Random biopsies taken from the duodenum to exclude celiac disease were negative.

Her colonoscopy showed an unusual nodular appearance in the distal colon (Figure 1). The mucosa also had an appearance similar to angiodysplasia. Multiple biopsies were taken from

\section{Pneumatose intestinale chez une patiente souffrant de bronchiectasie chronique}

La pneumatose intestinale a été décrite en lien avec de nombreux troubles digestifs, y compris l'infection, l'ischémie et l'obstruction. Elle a en outre été décrite chez des patients atteints de maladie pulmonaire obstructive chronique, de maladie des tissus conjonctifs, chez des receveurs de greffe d'organe, chez des patients atteints de leucémie et de divers types d'immunodéficience. Le présent article décrit le cas d'une femme de 66 ans souffrant de bronchiectasie chronique qui a éventuellement présenté une pneumatose intestinale.

this area, which were normal. Beyond $30 \mathrm{~cm}$, the remainder of the colon was normal.

The patient then underwent a double-contrast barium enema, which revealed very polypoid elevations throughout the sigmoid colon, and scattered similar lesions elsewhere. Several of these elevations were outlined by air, suggesting pneumatosis (Figure 2).

A computed tomography (CT) scan of the abdomen was then performed, followed by a CT colonography. These showed extensive intramural gas, mostly of a cyst-like configuration (Figures 3 and 4). The greatest involvement was in the sigmoid colon but it did extend down the rectum.

She was seen again in the clinic for follow-up and her continued symptoms. She was treated with Flagyl (sanofi-aventis Canada Inc) at $500 \mathrm{mg}$, three times per day for 10 days.

On discontinuation of Flagyl, she experienced worsening abdominal discomfort. She was then asked to continue taking Flagyl at $250 \mathrm{mg}$, twice per day.

Three months later, she reported that her abdominal discomfort and diarrhea improved greatly. She continued taking Flagyl for the next six months. Following this treatment, a repeat CT scan of her abdomen and a CT colonography were performed. These showed complete resolution of the PI (Figures 5 and 6).

\section{DISCUSSION}

PI can be defined as gas within the subserosal and/or submucosal layers of the bowel. It is an uncommon finding and the pathophysiology is uncertain.

\footnotetext{
${ }^{1}$ Department of Gastroenterology; ${ }^{2}$ Department of Radiology, The Ottawa Hospital, Ottawa, Ontario

Correspondence: Dr Maya Doumit, The Ottawa Hospital, 501 Smyth Road, Room LM10 - 501, Ottawa, Ontario K1H 8L6.

Telephone 613-737-8899 ext 76420, fax 613-232-8100, e-mail mayadoumit@hotmail.com

Received for publication January 22, 2008. Accepted April 10, 2008
} 

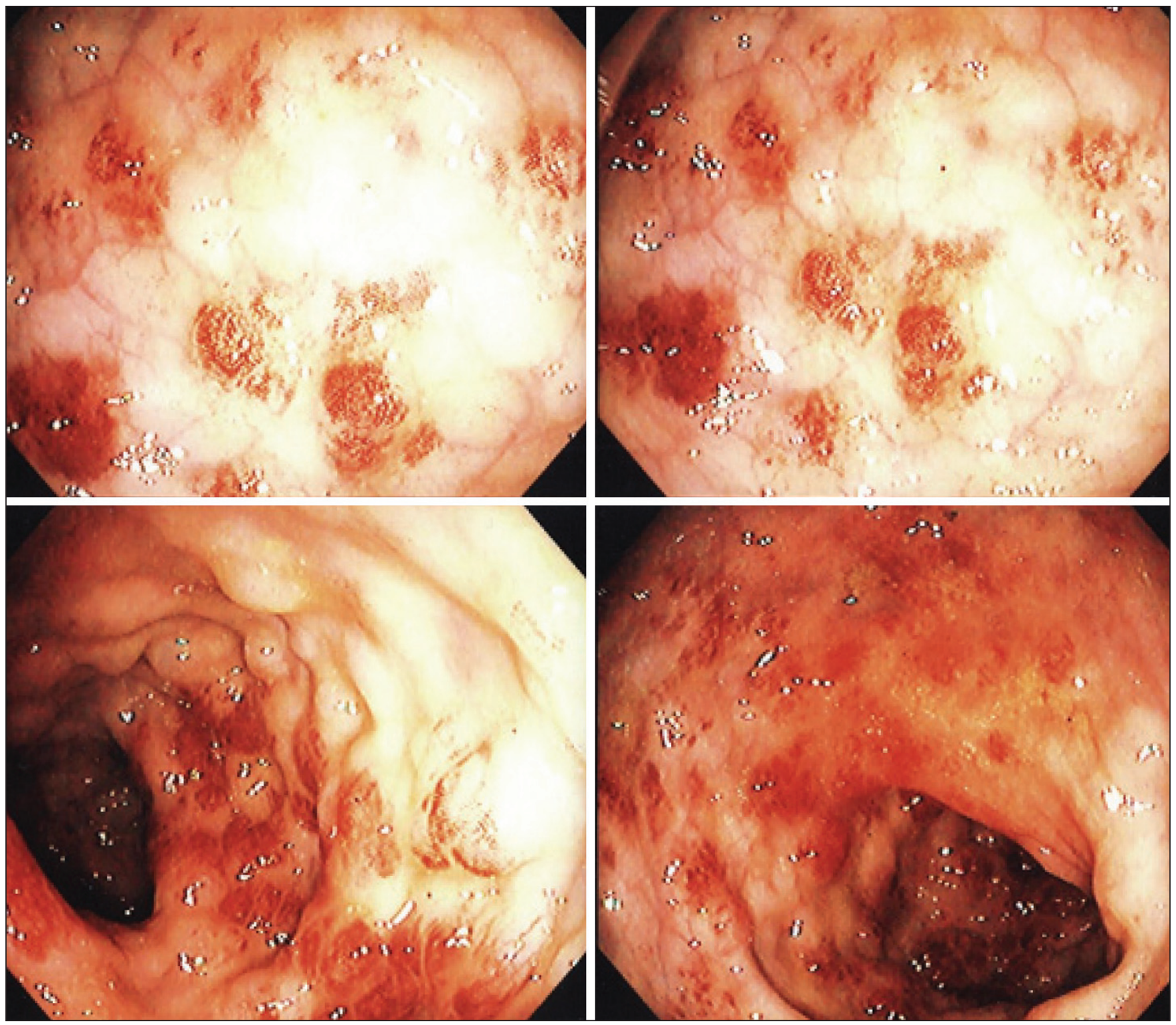

Figure 1) Colonoscopy, showing an unusual nodular appearance in the distal colon in keeping with pneumatosis intestinalis. These cystic lesions appear as a cluster of submucosal lesions ranging in size from a few millimetres to several centimetres. The mucosa also has an appearance similar to angiodysplasia

Three major theories seek to explain the pathogenesis of PI (5). One theory suggests that intraluminal gas makes its way into the bowel wall as a result of either increased intraluminal pressure (as with vomiting or obstruction), injury to the mucosa or its immune barrier (as with immunodeficiency states, therapy with corticosteroids or cytotoxic agents), or a combination of both increased pressure and mucosal compromise.

A second explanation implicates gut bacteria as a source of the intramural gas. Bacteria may invade a compromised mucosal barrier and produce intramural gas, or intraluminal bacteria may create high local concentrations of hydrogen, which subsequently diffuse into the bowel wall.

A third theory postulates that ruptured alveoli allow air to track along the vasculature, but this explanation has been met with skepticism due to the lack of interstitial air in the lung and mesentery of many patients with PI.

Chronic processes associated with pneumatosis include pulmonary disease (eg, chronic obstructive pulmonary disease, cystic fibrosis, artificial ventilation), inflammatory bowel disease, connective tissue disease (eg, scleroderma, systemic lupus erythematosus), immunocompromised states and Whipple's disease. Acute processes include ischemia caused by necrotizing enterocolitis, mesenteric vascular disease, colonic obstruction (air dissecting distally), trauma (eg, sigmoidoscopy, biopsy, barium enema, postsurgical anastomosis), infection (eg, primary infection, parasites, perforated jejunal diverticula) or inflammation. Less frequently, it may occur with no apparent associated disease ('primary' PI). For this reason, it is important to consider a patient's overall clinical status to discriminate between pathological and benign primary PI.

PI associated with ischemia has a mortality rate of approximately $50 \%$ to $75 \%$. Therefore, it is important to rule out ischemia when pneumatosis is seen.

The most common symptoms found in patients with pneumatosis are diarrhea, bloody stools, abdominal pain, abdominal distention, constipation, weight loss and tenesmus (6). 


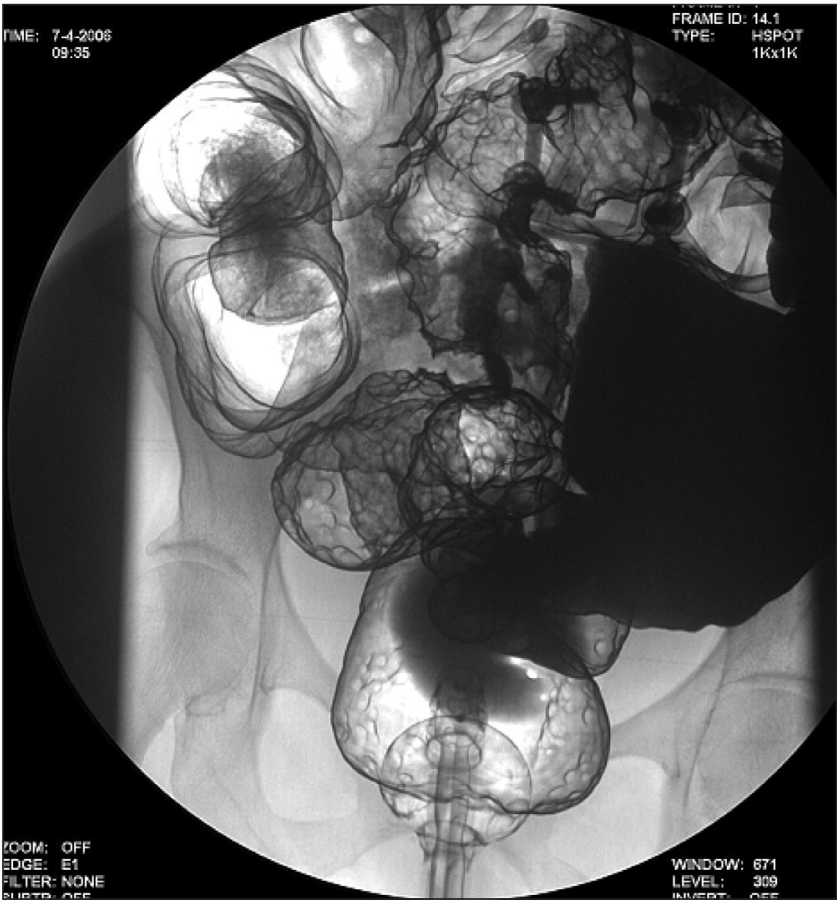

Figure 2) A double-contrast barium enema revealing very polypoid elevations throughout the sigmoid, and scattered similar lesions elsewhere. Several of these elevations are outlined by air, suggesting pneumatosis

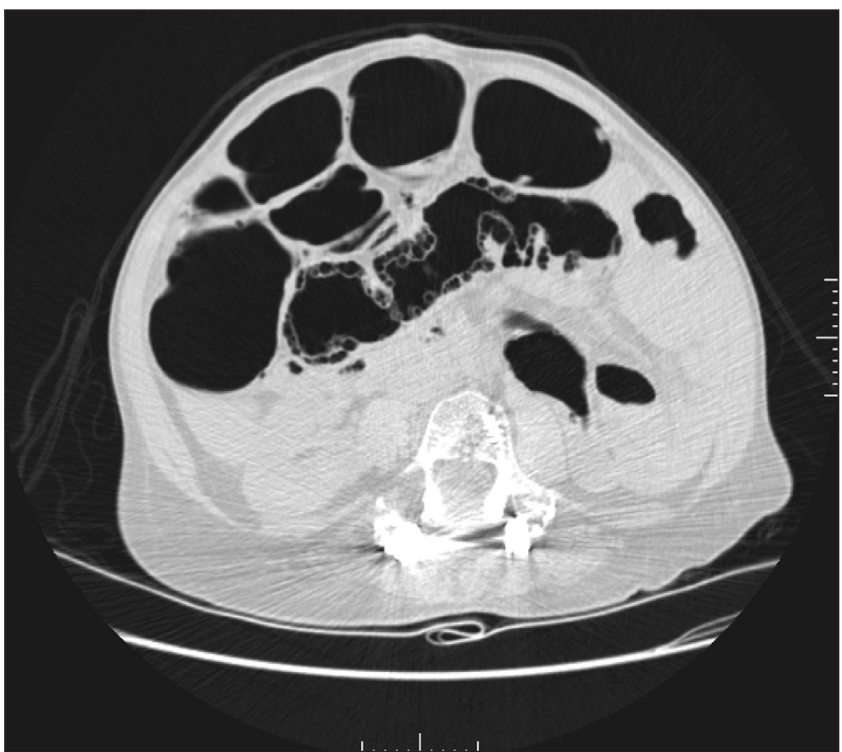

Figure 3) A transverse section of an abdominal computed tomography scan performed before treatment with Flagyl (sanofi-aventis Canada Inc), showing extensive cyst-like intramural gas collections parallel to the bowel wall, compatible with pneumatosis intestinalis. The greatest involvement was in the sigmoid colon, but it did extend down the rectum

Complications of pneumatosis coli occur in approximately $3 \%$ of patients and include pneumoperitoneum, volvulus, intussusception, hemorrhage and intestinal perforation (7). Review of the literature, however, revealed no complications secondary to colonic biopsy during the diagnosis of pneumatosis coli.

In asymptomatic patients, treatment is usually unnecessary because cysts resolve spontaneously in at least $50 \%$ of

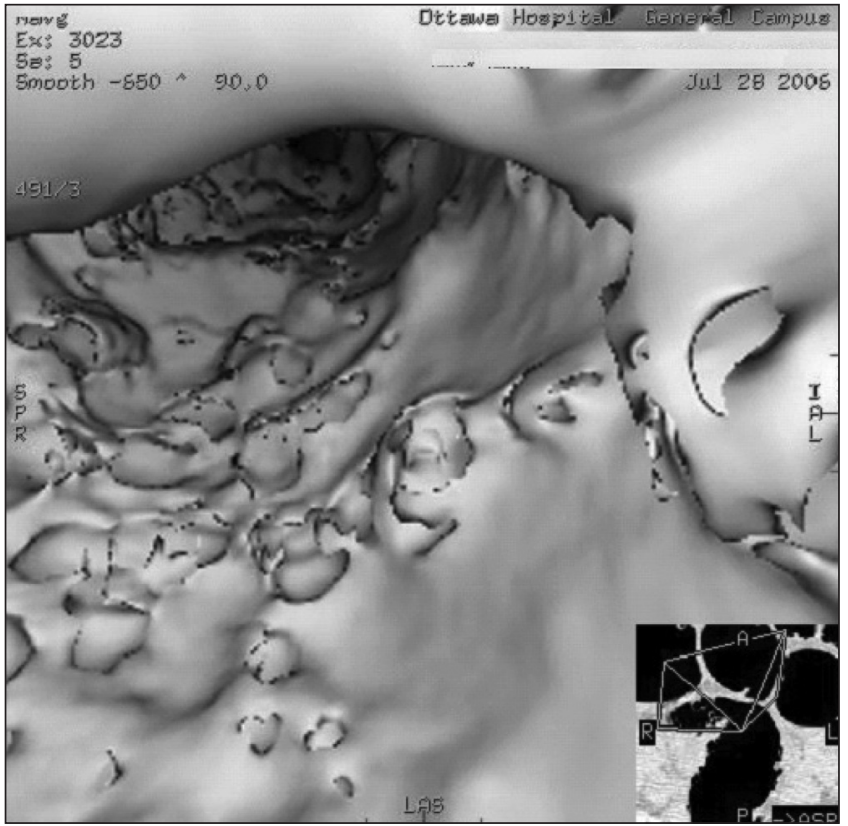

Figure 4) A computed tomography colonography performed before treatment with Flagyl (sanofi-aventis Canada Inc), showing extensive intramural gas, mostly of a cyst-like configuration compatible with pneumatosis intestinalis. The greatest involvement was in the sigmoid colon, but it did extend down the rectum
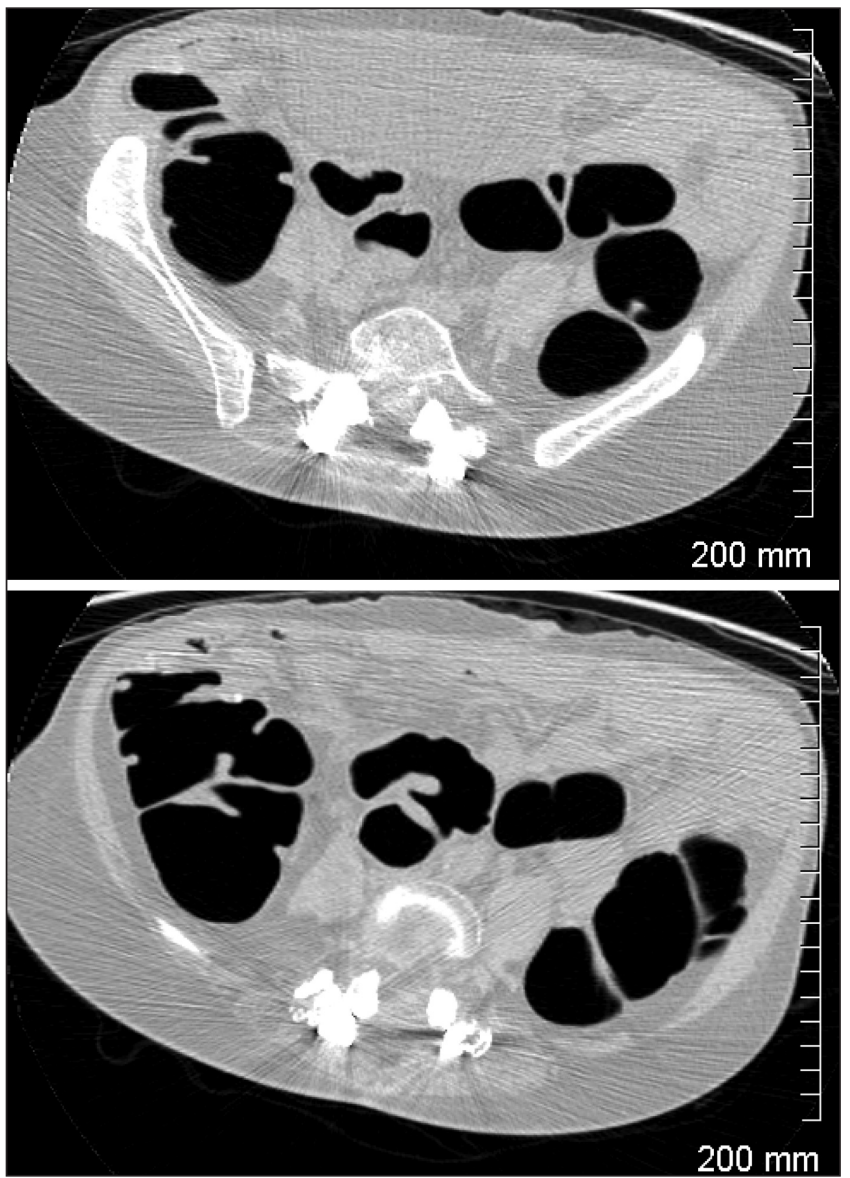

Figure 5) A transverse section of an abdominal computed tomography scan performed after treatment with Flagyl (sanofi-aventis Canada Inc), showing that the intramural gas parallel to the bowel wall completely disappeared. Only a few diverticula were identified 


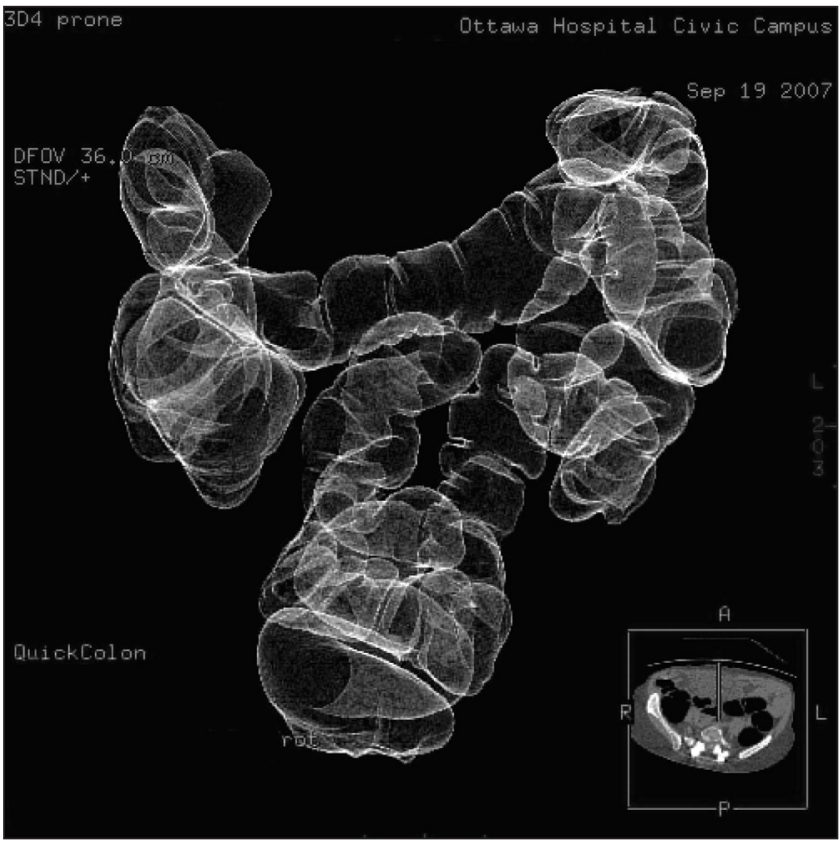

Figure 6) A computed tomography colonography performed one year after treatment with Flagyl (sanofi-aventis Canada Inc), showing complete resolution of the extensive pneumatosis intestinalis present on previous computed tomography colonography

patients (7). In patients with significant colonic symptoms, accelerated resolution of cysts and improvement in symptoms can follow treatment with elemental diet, antibiotics or the use of high-flow oxygen (Venturi face mask or hyperbaric oxygen) for several days. Flagyl $600 \mathrm{mg} /$ day to $1500 \mathrm{mg} /$ day in divided doses for up to several months has been reported as another treatment modality (8). For severe or refractory symptoms, surgery is usually required for patients with PI who remain symptomatic despite medical therapy or who develop complications from pneumatosis such as bowel obstruction. Although surgery can be effective, worsening of PI after surgery has also been observed (8).

Aside from chronic bronchiectasis, we cannot identify another explanation for our patient's pneumatosis. A recent
CT scan of the thorax showed diffuse bronchiectasis. A complete respirology assessment did not reveal an underlying etiology for her bronchiectasis.

PI in the setting of chronic bronchiectasis may be explained by the third theory discussed in the present paper, which suggests that blebs burst and the gas interpolates into the bowel wall. The trapped gas may be visible as 'bubbles' or 'bands'. The bubble appearance is generally considered to be early stage, with air bubbles trapped in the submucosa. The condition is potentially reversible. The band appearance manifests in transmural infarction, in which the gas is able to expand under the serosal surface.

Our search of the literature yielded no previous case reports of PI in the setting of chronic bronchiectasis. In the majority of cases, PI is a result of a variety of clinical conditions. Patients who do come to clinical attention can present in a variety of ways. In some cases, PI is an incidental finding, whereas in others, it portends a life-threatening intraabdominal condition. As a result of the diverse array of clinical settings in which PI is encountered, it can easily be missed without proper clinical vigilance. Therefore, educating physicians involved in the care of these patients is of paramount importance because it may help prevent misdiagnosis and unnecessary surgery.

\section{REFERENCES}

1. Mueller CF, Morehead R, Alter AJ, Michener W. Pneumatosis intestinalis in collagen disorders. Am J Roentgenol Radium Ther Nucl Med 1972;115:300-5.

2. Keats TE, Smith TH. Benign pneumatosis intestinalis in childhood leukemia. Am J Roentgenol Radium Ther Nucl Med 1974;122:150-2.

3. Andorsky RI. Pneumatosis cystoides intestinalis after organ transplantation. Am J Gastroenterol 1990;85:189-94.

4. Keinman PK, Brill PW, Winchester P. Pneumatosis intestinalis. Its occurrence in the immunologically compromised child. Am J Dis Child 1980;134:1149-51.

5. St Peter SD, Abbas MA, Kelly KA. The spectrum of pneumatosis intestinalis. Arch Surg 2003;138:68-75.

6. Jamart J. Pneumatosis cystoides intestinalis. A statistical study of 919 cases. Acta Hepatogastroenterol (Stuttg) 1979;26:419-22.

7. Liu KL, Chen HY, Lee TC, Wang HP. Gastrointestinal: Pneumatosis coli. J Gastroenterol Hepatol 2006;21:772.

8. Tak PP, van Duinen CM, Bun P. Pneumatosis cystoides intestinalis in intestinal pseudo-obstruction: Resolution after therapy with metronidazole. Dig Dis Sci 1992;37:949-54. 


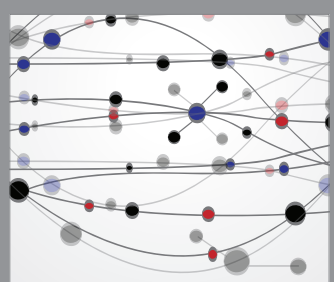

The Scientific World Journal
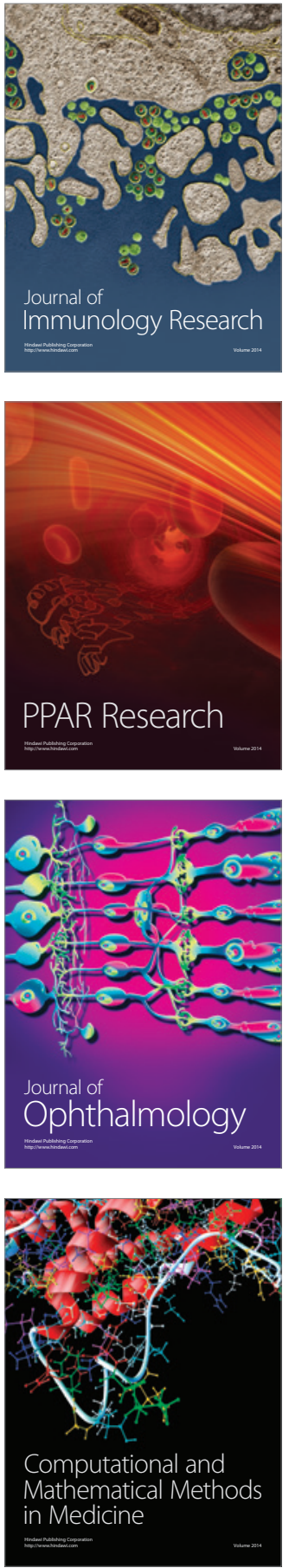

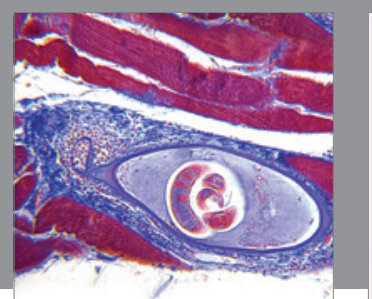

Gastroenterology Research and Practice

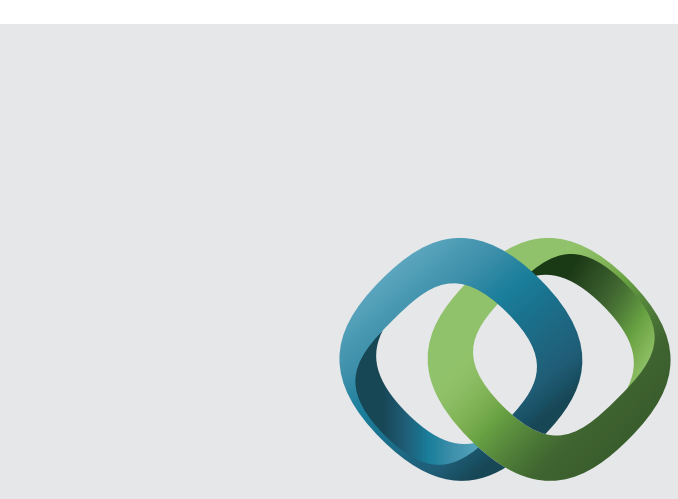

\section{Hindawi}

Submit your manuscripts at

http://www.hindawi.com
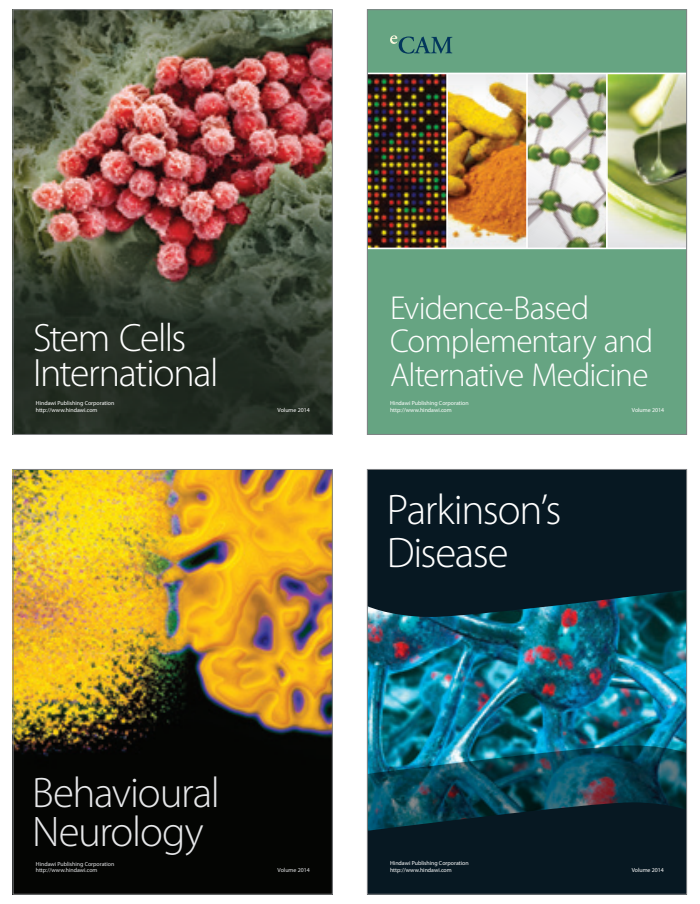
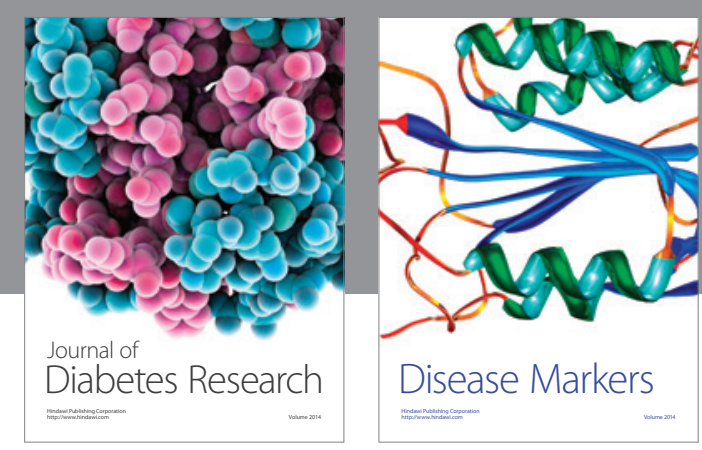

Disease Markers
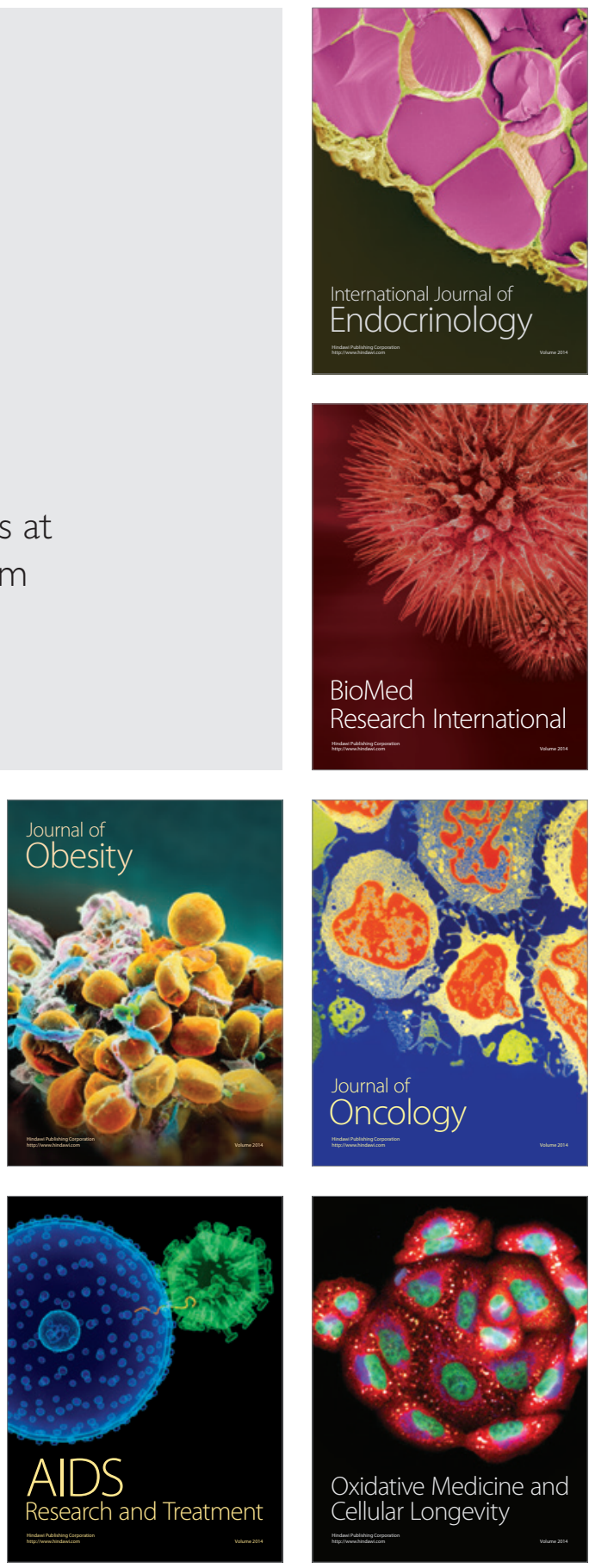